\title{
Vitamin D, muscle strength and physical function in UK South Asian older women
}

\section{Abstract}

A positive association has been reported between vitamin D status, muscle strength and physical function in older Caucasian women. However, little is known about this relationship in UK South Asian older women. This cross-sectional study aimed to explore the association between vitamin D status, muscle strength and physical function in UK South Asian (Pakistani and Indian) older women.

One hundred and twenty community-dwelling women were recruited via visiting community centres, mosques, Indian temples (Gurdwara) and by word of mouth (between January to May 2018). Inclusion criteria were: community-dwelling, South Asian women, age 60 years and above, able to communicate effectively and able to give written or verbal consent. A general interview (demographic, anthropometric, self-reported exhaustion, health history, supplementation use/duration/dose), handgrip strength $(\mathrm{kg})$, short physical performance battery (single chair stand, repeated chair stands, balance, timed up-and-go test), and blood 25 hydroxyvitamin $\mathrm{D}(25(\mathrm{OH}) \mathrm{D}, \mathrm{nmol} / \mathrm{l})$ concentration were performed. Dietary intake and self-reported physical activity was also assessed using multiple-pass 24-hour diet recall method and International Physical Activity Questionnaire (IPAQ) short form respectively.

Overall, $47 \%$ of participants had insufficient blood $25(\mathrm{OH}) \mathrm{D}$ concentration $(<50 \mathrm{nmol} / \mathrm{L})$ and $53 \%$ had sufficient blood $25(\mathrm{OH}) \mathrm{D}$ $(\geq 50 \mathrm{nmol} / \mathrm{L}$ ) using the IOM classification. As expected $86 \%$ of current vitamin D supplement users had adequate vitamin D status and only $14 \%$ of women who reported current vitamin D supplement use had insufficient vitamin D status. Amongst supplement non-users $68 \%$ had insufficient vitamin D status and only $32 \%$ had adequate levels. An inverse correlation (Spearman's analysis) was found between vitamin $\mathrm{D}$ status and single chair stand test $(\mathrm{sec})(\mathrm{r}=-0.25, \mathrm{p}=0.006)$; repeated chair stand test (sec) ( $\mathrm{r}$ $=-0.29, p=0.002)$ and timed up-and-go test $(\mathrm{sec})(\mathrm{r}=-0.20, \mathrm{p}=0.02)$. No correlation was observed between vitamin $\mathrm{D}$ status and handgrip strength $(\mathrm{r}=0.09, \mathrm{p}=0.30)$.

The prevalence of vitamin D insufficiency is high in post-menopausal South Asian women not taking vitamin D supplements. Significant association was observed between vitamin D status and some aspects of muscle strength and physical function. We are now conducting a randomized control trial to investigate whether vitamin D supplementation may restore muscle strength and function in this population.

\section{Conflict of Interest}

There is no conflict of interest 